\title{
The Society for Computer Applications in Radiology
}

$\mathbf{T}$ HE SOCIETY for Computer Applications in Radiology (SCAR) represents a unique opportunity to bring together in one forum individuals from both academics and industry who share a common interest in the development and application of computer techniques in radiology. Over the past 10 years, diagnostic imaging has become a critical component of the health care delivery system and, as a result, Radiology departments have become major elements of hospitals and outpatient clinics. As diagnostic imaging capabilities have expanded and radiology departments have grown, the need to manage them effectively has become a significant concern to both the department and to the hospitals they support. In addition, the ability to efficiently acquire diagnostic information, process it in the most effective manner to positively impact on patient care, and distribute it efficiently and effectively to bedside physicians is critically important to radiology departmental function. The solutions to these challenging problems can be best achieved by many individuals in both clinical medicine and industry working in concert to achieve practical solutions to complex problems. SCAR provides an excellent forum for the exchange of ideas in a meaningful and open fashion. Within the Society, Special Interest Groups (SIG) allow individuals to focus on areas of particular interest. Contact the SCAR office if you are interested in a SIG.

The SIG on Automated Radiology Reporting Systems was formed by SCAR to optimize efficiency and effectiveness in recording and communicating the interpretation of medical images; to foster the use of relevant technology; and to ensure that the timely contribution of the radiologist has maximum positive impact on the diagnosis and care of the patient. Chairman Alan H. Rowberg, MD, has established four subgroups: administrative/political, interfaces, survey of reporting practices, and documentation of resources.

Ronald B. Schilling, $\mathrm{PhD}$, is the chairman of the picture archiving communication system (PACS) Technology Forum special interest group of SCAR. This very active SIG met at the Radiologic Society of North America (RSNA) and also in February at the Society of Photo- optical Instrumentation Engineers (SPIE) Medical Imaging $\mathrm{V}$ Meeting. Its mission is "to provide knowledge and information on the clinical, technical, and financial effectiveness of PACS technology for the purposes of planning, assessment, and implementation. Well underway is the creation of a "PACS Handbook."

The Journal of Digital Imaging (JDI), which provides a vehicle through which peer-reviewed concepts and results may be rapidly disseminated to interested parties, is fundamental to the ultimate success of SCAR. The journal has been very positively received by individuals working in the field and continued growth is anticipated during the coming years. All SCAR members receive JDI, as it is the official journal of SCAR.

SCAR is proud to announce the upcoming Symposium on Computer Assisted Radiology, S/CAR 92 in Baltimore, MD on June 14-17, 1992. This conference will provide a forum for a broad audience of health care and computing professionals to assess the advancements and benefits of using digital computing for improving the clinical practice of radiology and biomedical sciences and to effectively manage health care resources. S/CAR 92 will be the 11th Conference on Computer Applications in Radiology.

SCAR serves to facilitate appropriate uses of computer applications in radiology and to enhance their further development. The benefits from these SCAR activities extend to everyone in radiology, but to realize the full benefits one needs to be a member of SCAR.

For membership information write to SCAR; PO Box 8800 , Harrisburg, PA $17105-8800$ or call $717-561-5266$. Membership is $\$ 125.00$ for the United States and Canada, $\$ 50.00$ for students and residents, and $\$ 155.00$ for all other countries. Membership does include the Journal of Digital Imaging, and the additional cost for members outside of the country relates entirely to handling and mailing costs for the journal.

$$
\begin{array}{r}
\text { Carl E. Ravin, MD } \\
\text { Chairman, SCAR } \\
\text { Department of Radiology } \\
\text { Duke University Medical Center } \\
\text { Durham, NC 27710 }
\end{array}
$$

